\title{
Pancreatic heterotopia in the gallbladder: an incidental finding after cholecystectomy
}

\author{
Maciej Sroczynski', Maciej Sebastian', Agnieszka Halon², Jerzy Rudnicki' ${ }^{1}$, \\ Agata Sebastian $^{3}$, Anil Kumar Agrawal ${ }^{4}$, Pawel Piekarz ${ }^{1}$
}

\author{
${ }^{1}$ Department of Minimally Invasive Surgery and Proctology, Wroclaw Medical University, \\ Wroclaw, Poland \\ ${ }^{2}$ Department of Pathomorphology and Oncological Cytology, Wroclaw Medical University, \\ Wroclaw, Poland \\ ${ }^{3}$ Department of Rheumatology and Internal Medicine, Wroclaw Medical University, Wroclaw, Poland \\ ${ }^{4} 2^{\text {nd }}$ Department and Clinic of General and Oncological Surgery, Wroclaw Medical University, \\ Wroclaw, Poland
}

\begin{abstract}
Ectopic pancreas in the gallbladder is found very rarely in histological examination after cholecystectomy. The etiology of this entity is not yet clear, but there exist several hypotheses about its origin. Our histological study revealed both exocrine and endocrine components of pancreatic tissue as shown by $\mathrm{H} \& \mathrm{E}$ and immunohistochemical staining of a gallbladder sections of a 55-year old man. Ectopic pancreatic tissue may be an underestimated cause of acute idiopathic pancreatitis thus detailed postoperative histological examination may decrease the number of acute pancreatitis cases without the known cause. (Folia Histochemica et Cytobiologica 2013, Vol. 51, No. 2, 174-177)
\end{abstract}

Key words: gallbladder, ectopic pancreas, gallstones, immunohistochemistry, cytokeratin, synaptophysin, chromogranin A

\section{Introduction}

Pancreatic heterotopia is defined as pancreatic tissue located outside the gland without any anatomical or vascular connection to the pancreas [1]. Ectopic pancreatic tissue in gastrointestinal tract is found in $0,6-13,7 \%$ of autopsy series and the most common localizations are stomach (25-60\%) and duodenum $(25-35 \%)$ [2]. Gallbladder is an extremely rare localization of pancreatic heterotopia and only thirty-three cases of this entity were described so far [3]. Macroscopically, it represents only small amount of

Correspondence address: M. Sebastian,

Department of Minimally Invasive Surgery and Proctology,

Wroclaw Medical University,

Borowska St. 213, 50-556 Wroclaw, Poland;

tel.: +48 7173430 00, mobil: +48 607868 096;

fax: +48717343009 ;

e-mail:mseba@op.pl foreign tissue within the gallbladder. Microscopic examination can detect pancreatic tissue and the presence of exocrine and/or endocrine components can be additionally confirmed by immunohistochemical (IHC) staining. Usually patients are asymptomatic but sometimes they can manifest clinical symptoms of cholecystitis, pancreatitis or even peritonitis $[3,4]$.

\section{Material and methods}

A 55-year old man without any relevant past medical history was admitted to the surgical department with symptoms of acute pancreatitis. Computed tomography (CT) revealed the signs of acute pancreatitis and several small concrements in the gallbladder with slight distension of bile ducts $(8 \mathrm{~mm})$. The patient was qualified to endoscopic retrograde cholangiopancreatography (ERCP). During ERCP the small concrements were removed and the endoscopic sphincterotomy was performed. Patient was discharged after 5 days without 


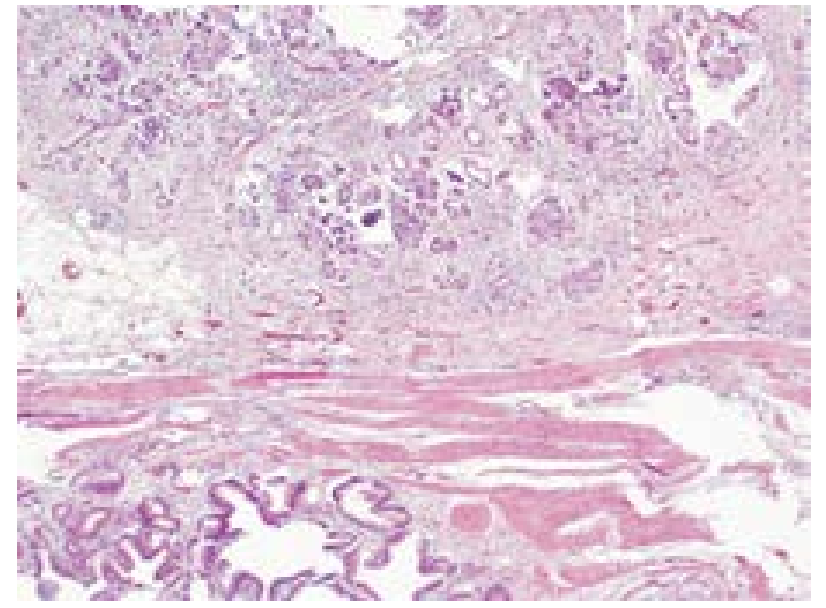

Figure 1. Microscopic appearance of the heterotopic pancreatic tissue located in the gallbladder wall. $H \& E(\times 40)$

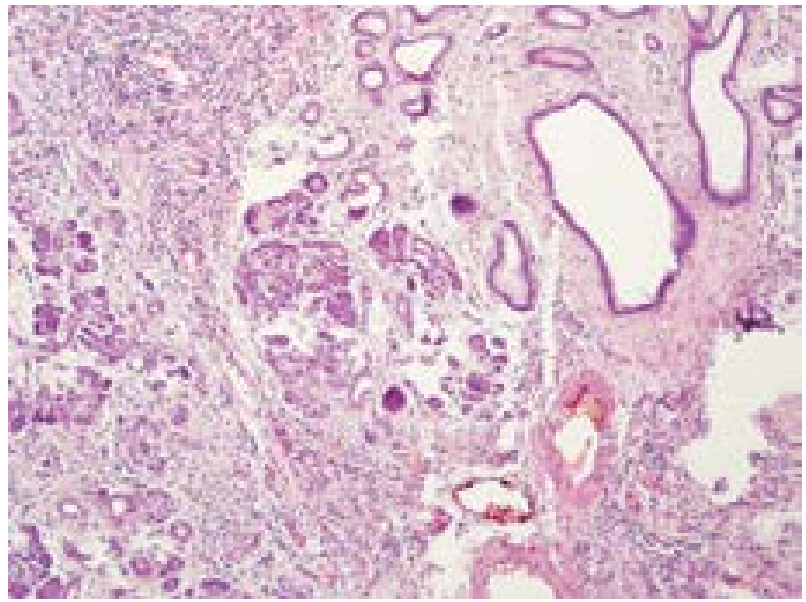

Figure 2. Histologic study of the gallbladder showing ectopic pancreatic tissue. Type I according to Heinrich's classification. H\&E $(\times 200)$
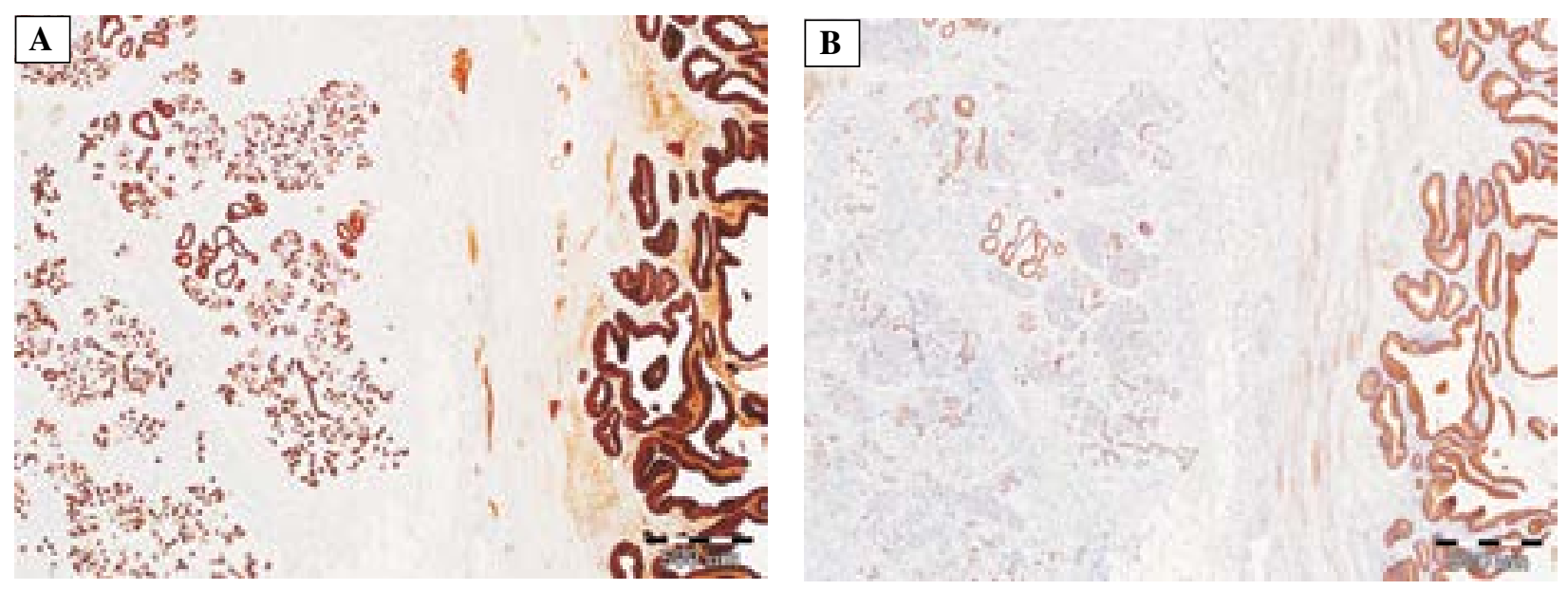

Figure 3. Positive staining for cytokeratin 7 (A) and cytokeratin 19 (B) in normal gallbladder epithelium (right side of the section) and ducts within acini of ectopic pancreas (left side of the section) $(\times 40)$

any complications. After 21 days he was admitted for the second time for laparoscopic cholecystectomy. Conversion to an open procedure had to be done due to strong adhesions of the gallbladder with greater omentum, right colic flexure and pylorus. The intraoperative examination revealed a $7 \mathrm{~cm}$ long gallbladder with thickened, green-yellow wall and small concrements inside. Patient was discharged 3 days after surgery without any complications. The operation was curative and he remains asymptomatic until now.

Histological sections were prepared and examined by histopathologist. Formalin-fixed, paraffin-embedded and hematoxilin and eosin (H\&E)-stained sections were analyzed. Additionally, an IHC examination was performed on the paraffin sections, evaluating positive expressions of cytokeratin (CK) 7 and cytokeratin (CK) 19 in ducts within acini of ectopic pancreas and synaptophysin and chromogranin A in islets of Langerhans. Antibodies used in the IHC assay were acquired from DAKO corporation. Primary antibodies were delivered in a ready-to-use/prediluted form (mouse anticytokeratin 7 clone OV-TL 12/30, anti-cytokeratin 19 clone RCK 108 and anti-synaptophysin clone DAK-SYNAP), and the visualization system EnVision ${ }^{\mathrm{TM}}$ FLEX + (DAKO) in high $\mathrm{pH}$ was used. The IHC technique was automatically performed in an Autostainer Link48 (DAKO).

\section{Results and discussion}

Microscopic examination revealed chronic cholecystitis and a $7 \mathrm{~mm}$-wide area of heterotopic pancreatic tissue within the gallbladder wall in fundus area (Figures 1 and 2). IHC studies confirmed the presence of both exocrine components with positive reactions for CK 7 (Figure 3A) and CK 19 (Figure 3B) and endocrine components with positive reactions for synaptophysin (Figure 4A) and chromogranin A (Figure 4B). 

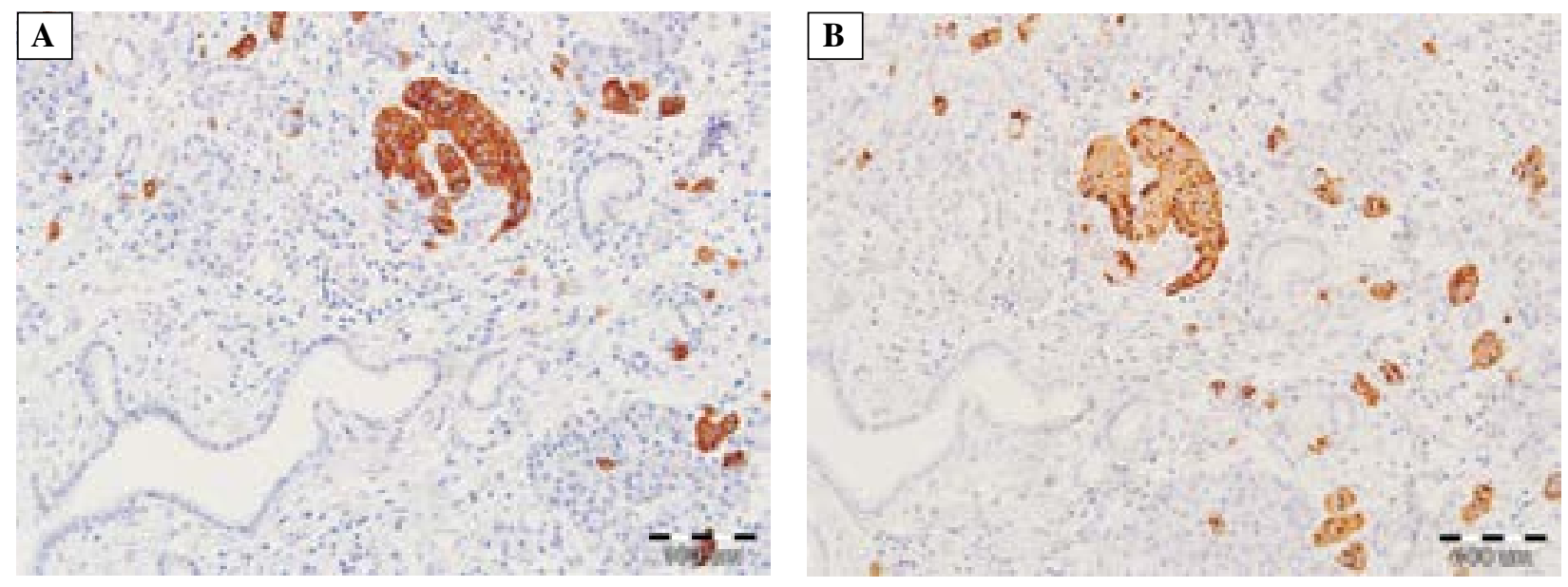

Figure 4. Cells in small pancreatic islets show immunoreactivity for synaptophysin (A) and chromogranin A (B). This immunohistochemical staining pattern is consistent with the endocrine component and type I heterotopia according to Heinrich $(\times 200)$

The etiology of pancreatic heterotopia is not yet clear but there are three hypotheses about its origin. The first one suggests the separation of heterotopic pancreatic tissue from the primitive pancreas during embryonic rotation. The second one suggests that pancreatic tissue is abnormally transported by the longitudinal growth of the intestines from lateral budding of the rudimentary pancreatic duct while penetrating the intestinal wall $[5,6]$. There also exists a theory based on abnormalities in the Notch signaling system, that lead to changes in differentiation in the developing foregut endoderm [7].

According to Heinrich's classification there are three types of ectopic pancreas. Type 1 has both exocrine and endocrine components, type 2 contains exocrine pancreatic tissue but without islet cells and type 3 with only pancreatic ducts present. It was observed that $50 \%$ of ectopic tissue does not contain islet cells [8].

In contrary to other reports which stated that pancreatic tissue in the gallbladder was more common in women and asymptomatic $[9,10]$, our patient was male and had symptoms of acute pancreatitis. We agree with other authors that it is impossible to diagnose pancreatic heterotopia in the gallbladder before and during operation and only very detailed postoperative histological examination can detect pancreatic tissue. Immunohistochemical study which localizes cells positive for the presence of CK7, CK19, synaptophysin and chromogranin A, may help to detect exocrine and endocrine components of heterotopic pancreatic tissue. Only a few authors used immunohistochemistry for detection of pancreatic heterotopia finding tubular and epithelial structures which showed immunopositive reactions to $\mathrm{CK} 7,8,18,19$ and to CA $19-9[3,5,11]$. Additionally, exocrine activity was confir- med by immunoreactivity to alpha-1-antitrypsin and alpha-1-chymotrypsin [3]. The presence of islet cells was confirmed by immunoreactivity to synaptophysin and chromogranin A [11]. Insulin and somatostatin may be other IHC markers for identifying endocrine activity [11]. The gallbladder and the pancreas have the same embryologic origin and unequivocal differentiation between pancreatic and gallbladder epithelium on the basis of specific markers is impossible. It has been suggested that proliferating pancreatic duct cells express vimentin [12], but further studies did not confirm positive reactions $[3,11]$.

So far, neither ultrasound nor CT and magnetic resonance imaging can confirm the diagnosis of ectopic pancreas preoperatively $[1,9,10]$. Differential diagnosis based on the presence of gallstones, cholesterol polyps, adenomyoma, carcinoma, cholecystitis, cysts, abscess formation and other lesions is almost impossible [9]. Radical excision is the treatment of choice in pancreatic heterotopia and is aimed at avoiding potential complications [4, 13]. Active pancreatic enzymes secreted into the gallbladder and biliary tract may lead to chronic inflammation, hyperplasia and dysplasia of the gallbladder mucosa and even to carcinoma of gallbladder [11, 14]. It has been suggested that pancreatic heterotopia may also be the underlying cause of other cancers, e.g. intraductal papillary mucinous neoplasm in jejunum [15].

We conclude that ectopic pancreatic tissue in the gallbladder should receive more attention as the possible cause of acute idiopathic pancreatitis. The limited data on this topic does not allow us to make general conclusions, however, it should be stressed that only strict cooperation between surgeon and histopathologist may reveal more cases of this entity. 


\section{References}

1. Distler M, Rückert F, Aust D, Saeger HD, Grützmann R. Pancreatic heterotopia of the duodenum: anatomic anomaly or clinical challenge? J Gastrointest Surg. 2011;15:631-636.

2. Neupert G, Appel P, Braun S, Tonus C. Heterotopic pancreas in the gallbladder. Diagnosis, therapy, and course of a rare developmental anomaly of the pancreas. Chirurg. 2007;78:261-264.

3. Klimis T, Roukounakis N, Kafetzis I, Mouziouras V, Karantonis I, Andromanakos N. Heterotopic pancreas of the gallbladder associated with chronic cholecystitis and high levels of amylasuria. J Pancreas (Online) 2011;12:458-460.

4. She WH, Cheung TT, Ng KK. A rare cause for peritonitis: pancreatic heterotopia. Int J Colorectal Dis. 2011;26:677-678.

5. Pilloni L, Cois A, Uccheddu A, Ambu R, Coni P, Faa G. Complete pancreatic heterotopia of gallbladder with hypertrophic duct simulating an adenomyoma. World J Gastroenterol. 2006;12:1786-1787.

6. Elpek GO, Bozova S, Küpesiz GY, Oğüş M. An unusual cause of cholecystitis: heterotopic pancreatic tissue in the gallbladder. World J Gastroenterol. 2007;13:313-315.

7. Artavanis-Tsakonas S, Rand MD, Lake RJ. Notch signaling: cell fate control and signal integration in development. Science. 1999;284:770-776.
8. Zinkiewicz K, Juskiewicz W, Zgodzinski W et al. Ectopic pancreas: endoscopic, ultrasound and radiological features. Folia Morphol (Warsz). 2003;62:205-209.

9. Cerullo G, Marrelli D, Di Mare G et al. Heterotopic pancreatic tissue in the gallbladder. Two case reports and brief review of the literature. G Chir. 2011;32:259-262.

10. Kondi-Paphiti A, Antoniou AG, Kotsis T, Polimeneas G. Aberrant pancreas in the gallbladder wall. Eur Radiol. 1997;7:1064-1066.

11. Beltrán MA, Barría C. Heterotopic pancreas in the gallbladder: the importance of an uncommon condition. Pancreas. 2007;34:488-491.

12. Ko SH, Suh SH, Kim BJ et al. Expression of the intermediate filament vimentin in proliferating duct cells as a marker of pancreatic precursor cells. Pancreas. 2004;28:121-128.

13. Kattepura S, Nanjegowda NB, Babu MK, Das K. Macroscopic pancreatic heterotopia on a congenital biliary dilatation. Pediatr Surg Int. 2010;26:847-849.

14. Seok WL, Sung PY, Hyung-Il S. Heterotopic pancreas of the gallbladder associated with segmental adenomyomatosis of the gallbladder. J Korean Surg Soc. 2013;84:309-311.

15. Okamoto H, Fujishima F, Ishida K et al. Intraductal papillary mucinous neoplasm originating from a jejunal heterotopic pancreas: report of a case. Surg Today. 2013; DOI 10.1007/s00595-012-0486-0.

Submitted: 18 January, 2013 Accepted after reviews: 13 June, 2013 